Astrophysical Journal Letters, unedited preprint

Preprint typeset using $\mathrm{LAT}_{\mathrm{E}} \mathrm{X}$ style emulateapj v. 08/22/09

\title{
THE BRIGHTNESS OF DENSITY STRUCTURES AT LARGE SOLAR ELONGATION ANGLES: WHAT IS BEING OBSERVED BY STEREO/SECCHI?
}

\author{
N. Lugaz $^{1}$, A. Vourlidas ${ }^{2}$, I. I. Roussev ${ }^{1}$, C. Jacobs ${ }^{3}$, W. B. Manchester, IV ${ }^{4}, \mathrm{O}$ Cohen $^{4}$ \\ Received 2008 May 23; accepted 2008 July 31
}

\begin{abstract}
We discuss features of coronal mass ejections (CMEs) that are specific to heliospheric observations at large elongation angles. Our analysis is focused on a series of two eruptions that occurred on 2007 January 24-25, which were tracked by the Heliospheric Imagers (HIs) onboard STEREO. Using a three-dimensional (3-D) magneto-hydrodynamic simulation of these ejections with the Space Weather Modeling Framework (SWMF), we illustrate how the combination of the 3-D nature of CMEs, solar rotation, and geometry associated with the Thomson sphere results in complex effects in the brightness observed by the HIs. Our results demonstrate that these effects make any in-depth analysis of CME observations without 3-D simulations challenging. In particular, the association of bright features seen by the HIs with fronts of CME-driven shocks is far from trivial. In this Letter, we argue that, on 2007 January 26, the HIs observed not only two CMEs, but also a dense corotating stream compressed by the CME-driven shocks.

Subject headings: scattering - MHD — Sun: corona - Sun: coronal mass ejections (CMEs)
\end{abstract}

\section{INTRODUCTION}

Coronal Mass Ejections (CMEs), one of the most extreme events in the solar system, have been studied extensively for over three decades now (see reviews by Schwenn et al. 2006; Roussev \& Sokolov 2006). Until the launches of the Solar Mass Ejection Imager (SMEI) (Evles et al. 2000) and the Solar Terrestrial Relations Observatory (STEREO) (Kaiser et al. 2008) in 2003 and 2006, respectively, continuous observations of CMEs were made by the $\mathrm{SoHO} / \mathrm{LASCO}$ coronagraphs out to a radial distance from the Sun of $32 R_{\odot}$.

The Heliospheric Imagers (HI-1 and HI-2, or collectively HIs) (Harrison et al. 2005), part of the SunEarth Connection Coronal and Heliospheric Investigation (SECCHI) suite onboard STEREO (Howard et al. 2008), enable the tracking of CMEs up to elongation angles (i.e. angular distance from the Sun) of $90^{\circ}$. These instruments observe the photospheric light scattered by free electrons in the solar corona and heliosphere (Thomson scattering, e.g. see Minnaert 1930). The scattering strongly depends on the position of the observer relative to the radial direction of the observed electron. The scattering is maximized, along a given line of sight (LOS), at the point of closest approach to the Sun; namely, when the LOS is normal to the radial direction of the electron. The locus of these points for all possible LOS forms a sphere, called the Thomson sphere (TS) by Vourlidas \& Howard (2006), whose center is the halfway point between the Sun and the observer and whose diameter is equal to the Sun-observer

\footnotetext{
${ }^{1}$ Institute for Astronomy, University of Hawaii, 2680 Woodlawn Dr., Honolulu, HI 96822; nlugaz@ifa.hawaii.edu, iroussev@ifa.hawaii.edu

${ }^{2}$ Code 7663, Naval Research Laboratory, Washigton, DC 20375; vourlidas@nrl.navy.mil

${ }^{3}$ Centrum voor Plasma Astrofysica, KU Leuven, Celestijnenlaan 200B bus 2400, 3001 Leuven, Belgium ; carla.jacobs@wis.kuleuven.be

${ }^{4}$ Department of AOSS, University of Michigan, 2455 Hayward St., Ann Arbor, MI 48198; chipm@umich.edu;oferc@umich.edu
}

distance. Jackson \& Leinert (1985) applied this concept to zodiacal photometric observations made by Helios at heliospheric distances as close as 0.31 AU. Recently, Vourlidas \& Howard (2006) examined the influence of the TS for the analyses of CMEs observed by the HIs and SMEI by studying the evolution of the brightness of simple structures at large elongation angles.

Additionally, the progenitors of Corotating Interaction Regions (CIRs), which are dense streams associated with the interaction of a fast stream of the solar wind originating from a coronal hole with the slow solar wind, have been imaged out to Earth's orbit by the HIs (Sheelev et al. 2008b). Sheeley et al. (2008a) and Rouillard et al. (2008) have described how the elongation versus time tracks of the dense streams show apparent accelerations/decelerations depending on the distance of the structures from the observer. These studies suggest the need for a revision of the standard paradigm of tracking structures in the corona to adapt it to density structures propagating in the inner heliosphere. However, these revisions alone are not sufficient to understand in detail the evolution of CMEs as they propagate in the HIs' FOVs. CMEs are intrinsically $3-\mathrm{D}$ density structures which, compared to CIRs, have a much larger and varying width and can be rather asymmetric. The visibility of CME structures is, therefore, subject to geometric and Thomson scattering effects that may be hard to distinguish from evolution or propagation effects. To make progress, fully 3-D numerical simulations of CME evolution in the heliosphere associated with synthetic LOS capabilities are important (Odstrcil et al. 2005; Lugaz et al. 2005; Aschwanden et al. 2008; Sun et al. 2008).

Manchester et al. (2008) studied the evolution of CME brightness in the HI-2's FOV and predicted two features due to the effects of the TS: (i) the apparent deceleration (called "stall" by the authors) of a CME front observed in HI-2 (similar to CIR observations by STEREO-A) and, (ii) the appearance of a "second" front due to multiple 
crossings of the TS by the CME. However, their study was for an extremely dense and fast CME (part of the Halloween storm); this causes the solar wind background to be less important than for slower CMEs. In this Letter, we go further by analyzing a complex series of events, one actually observed by STEREO: the 2007 January 2425 CMEs interacting in the heliosphere. These were the first major CMEs detected by the HIs, and they have been described by Harrison et al. (2008). The 3-D modeling has been done using the Space Weather Modeling Framework (SWMF) (\$2). Here, we focus on the influence of the TS in determining what density structures have been observed, focusing on HI-2 observations on 2007 January 26 (\$3). The final conclusions of this investigation are drawn in 4

\section{THE SOLAR ERUPTIONS OF 2007 JANUARY 24-25}

\subsection{Coronagraphic and Heliospheric Observations}

On 2007 January 24, at 1403UT, COR1 (FOV: 1.5$4 R_{\odot}$ ) observed an eruption from active region (AR) 940, which was behind the eastern limb of the Sun at the time. At 0643UT on January 25, COR1 observed a second eruption from the same AR. These two eruptions were also detected by SoHO/LASCO. Based on timeheight profiles in the LASCO FOVs, they had a projected speed between 600 and $750 \mathrm{~km} \mathrm{~s}^{-1}$ for the first CME, and between 1,000 and 1,350 $\mathrm{km} \mathrm{s}^{-1}$ for the second one, depending on the position angle (PA, angle from the Solar North towards Solar East) of the measurement. Using these speeds derived from near-Sun observations and assuming that there was no deceleration, the two CMEs were expected to interact on January 25 at 2030UT near PA $80^{\circ}$ at a radial distance of $97 R_{\odot}(0.45 \mathrm{AU})$. Further assuming that both CMEs occurred exactly on the east limb, the corresponding elongation angle would be $25^{\circ}$ (in HI-2's FOV).

At the time of the ejections, being in the commissioning phase of the mission, STEREO-A was rolled by $22.4^{\circ}$ from the solar north, which resulted in HI-1 and HI-2 imaging higher-latitude regions than during the normal phase of the mission (Harrison et al. 2008). The spacecraft was separated by approximately $0.4^{\circ}$ from Earth and was at a radial distance of 0.97 AU from the Sun. The leading ejection was first detected in HI-1's FOV at $1801 \mathrm{UT}$ on January 24 at $4.4^{\circ}$ elongation and was tracked until $12.1^{\circ}$ at $0401 \mathrm{UT}$ on January 25 . At that time, there was a SECCHI data gap for 20 hours. Multiple fronts were detected in HI-2 on January 26: the brightest one propagated from $24.7^{\circ}$ at $0201 \mathrm{UT}$ to $32.5^{\circ}$ at 1801UT. Howard et al. (2008) noted the presence of "forerunner" structures (at $42^{\circ}$ at 1801UT). Even after accounting for the deceleration of the CMEs, these observations are likely to have covered the interaction of the two successive CMEs. The ability of the HIs to detect CME mergers in the heliosphere has been predicted by Lugaz et al. (2008). The difficulty, however, is to associate the structures observed in HI-2 to the individual ejections.

\subsection{Simulation Set-up}

The 3-D simulation of the two ejections was done using the SWMF (Tóth et al. 2005). The solar corona domain of SWMF is resolved with 40,489 blocks of $4^{3}$ cells each, ranging in size from $1 / 40 R_{\odot}$ at the inner boundary to $0.75 R_{\odot}$ at the outer boundary. The inner heliosphere domain of SWMF is resolved with 16,626 blocks of $8^{3}$ cells each, ranging in size from 3.44 to $0.215 R_{\odot}$. In both domains, the heliospheric current sheet is refined in order to better capture the density gradients there. The solar wind and coronal magnetic field are reproduced using the model developed by Cohen et al. (2007). This model makes use of solar magnetogram data and the Wang-Sheeley-Arge (WSA) model (Wang \& Sheeley 1990; Arge \& Pizzo 2000) for the asymptotic solar wind speed at $1 \mathrm{AU}$. The solar magnetic field is reconstructed from a Legendre polynomial expansion of order 49 based on NSO/SOLIS magnetogram data.

To model the CMEs, we use a semi-circular flux rope prescribed by a given total toroidal current, as in the models by Titov \& Démoulin (1999) and Roussev et al. (2003). A more complete description of the implementation of this model can be found in Lugaz et al. (2007). This flux rope solution, once superimposed on the background magnetic field, leads to immediate eruption because of force imbalance The flux rope parameters are chosen such that there is an agreement with the observed values of the speed of the eruptions in the corona. Our main goal here is to study the interaction of the two ejections with the background solar wind and with each other.

\section{TWO CMES AND A DENSE STREAM}

\subsection{Goal of the Study}

The STEREO spacecraft have great potential to enhance the quality of space weather predictions, because density structures can be tracked on their way to Earth beyond LASCO FOV (Kaiser et al. 2008). However, one of the difficulties associated with the interpretation of SECCHI/HIs observations stems from the need to distinguish between different types of density structures in the heliosphere. During the first year of the mission, observations focused on CIRs and CMEs, which have been easily observed at large elongation angles. There has not been any reported observation of CMEs interacting with CIRs in the HI-2's FOV yet. This relative simplicity of HI-2's observations should change as we approach the more active phase of solar cycle 24. Effects such as those discussed by Vourlidas \& Howard (2006), Manchester et al. (2008) and Rouillard et al. (2008) should make the direct association of bright fronts observed by the HIs with ejections observed by coronagraphs more complicated. In this section, we show how the knowledge gained from the careful analysis of 3-D simulations can significantly improve the interpretation of HI observations.

On January 26, HI-2 onboard STEREO-A observed a number of structures associated with the CMEs of 2007 January 24-25 (Fig. 1, left panel). Here, we focus on the observations and model results at 0600UT on January 26 to try to determine the origin of these structures. This particular time was chosen because three distinct structures were clearly visible in HI2's image. In this analysis, we analyze running difference images, the most common type of imaging used to track faint structures far from the Sun.

\subsection{The Two Ejections}



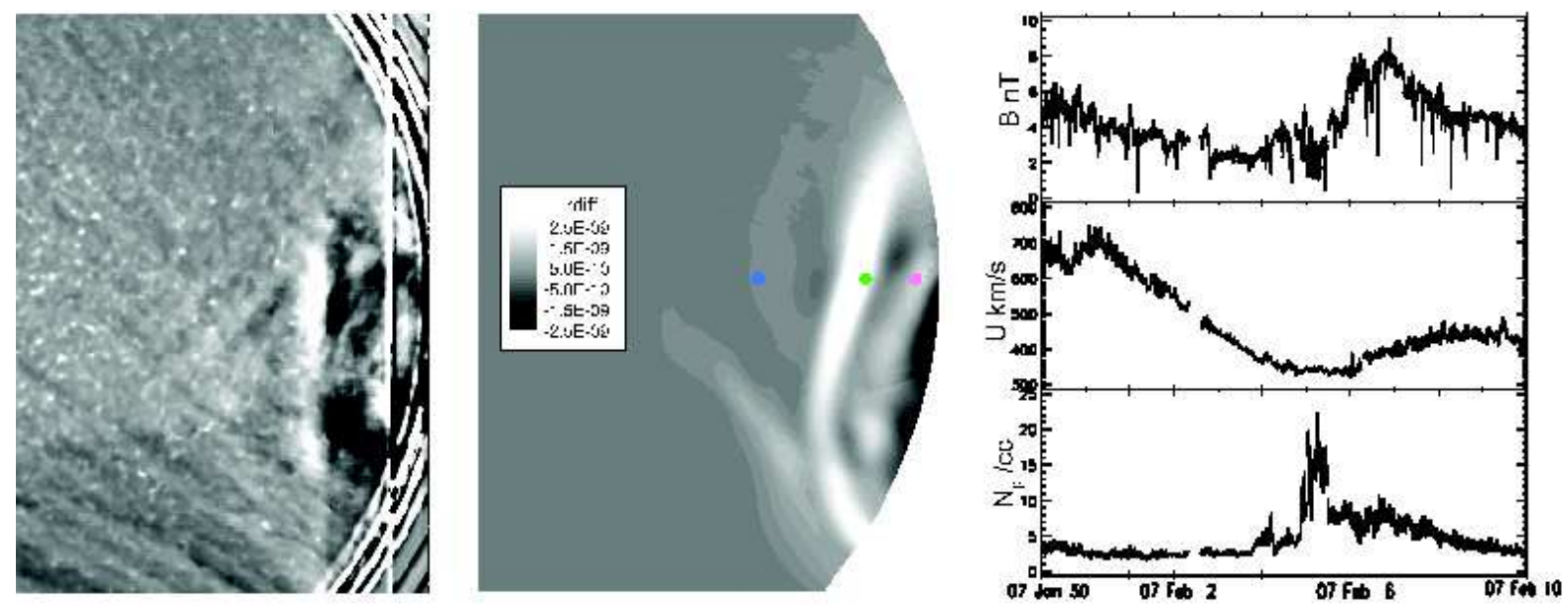

FIG. 1.- Left: SECCHI HI-2A running difference image at 0600UT on 2007 January 26. Middle: Simulated SECCHI HI-2A running difference image at 0600UT on 2007 January 26. The dots correspond to elongations of $30^{\circ}, 23^{\circ}$ and $20^{\circ}$ from left to right. Right: Wind observations during this time period showing a dense stream hitting the Earth on 2007 Feb 5, corresponding to the structure marked with the pink dot.

In the right panel of Fig. 2, we show a two-dimensional cut of the simulation results as seen from the solar north pole (showing the density scaled by $1 / \mathrm{R}^{2}$ ) at $0600 \mathrm{UT}$ on January 26 with the schematic of the observations. As can be seen in this image, the two CMEs have partially merged, but there are still two distinct fronts in the vicinity of the Sun-Earth line. In our synthetic HI2 image (Fig. 1, middle panel), there are two bright structures (blue and green dots) associated with these two distinct ejections. The second front (green dot) is brighter because it corresponds to material swept up with the first ejection which has been additionally compressed by the second ejection. This enhanced density is often observed in sheaths associated with interacting CMEs (Farrugia et al. 2006; Lugaz et al. 2008).

The protruding front in the southern half of the synthetic HI-2 image (also present, dimly, in the real HI-2 image) is also associated with the leading edge of the first CME (blue dot). As seen in halo CMEs near solar minimum, the leading edge propagates faster in highlatitude regions, where the Alfvénic speed is higher and the density is lower than near the current sheet. Because STEREO-A was rolled by $22.4^{\circ}$ from the solar north, the center of the LOS images corresponds to mid-latitude regions. This roll enhances the apparent protrusion of the first front.

Following this analysis, we associate the brightest structure in the real HI-2 image with the CME on 2007 January 25 , which is in the process of overtaking the previous CME. We believe that the fainter structure ahead corresponds to the first CME. We will now focus on the origin of the third structure marked with a pink dot in the middle panel Fig. 1.

\subsection{The Dense Stream $(D S)$}

In the left panel of Fig. 2, we show the running difference of the 3-D density on the Thomson sphere and the plane corresponding to the center of the LOS image $\left(\mathrm{PA} 67.6^{\circ}\right)$. This is an approximate way to visualize the results of the simulation in a manner similar to the HI2 images. The three structures are marked with three LOS rays which correspond to the dots of the same color in the middle panel of Fig. 1. Note that the Thomson scattering decreases quite slowly away from the TS (Vourlidas \& Howard 2006). Therefore, density enhancements away from the TS should be considered, but are hard to visualize (see online animation). Looking at the simulation result, we have found that the pink dot is associated with a density increase on the TS distinct from the two CME fronts, but the ray also intersects the second CME. After careful analysis of the simulation results, we have determined that this distinct density increase is likely associated with a dense corotating stream (DS), which has been compressed by the two CMEs (see Fig. 2, right).

According to our simulation, the DS was present in the heliosphere prior to the CME passage ("DS" in the right panel of Fig. 2), with a density about 50\% larger than the average density at the same heliospheric distance. The simulation shows how the DS is compressed by the two CMEs (right panel of Fig. 2), and due to co-rotation it enters the FOV of HI-2 in January 26. The presence of this density enhancement prior to the passage of the CMEs and the small angular span of this denser region clearly rules out the possibility that this increased density is associated with the CMEs. Our results indicate that, in the absence of any CMEs, the Earth would have passed a region of enhanced density between 0900UT on Feb 4 and 0100UT on Feb 6 (peak density at 1700 UT on Feb 4). In reality, a DS was observed at the Earth on Feb 5, as seen in the Wind data shown on the right panel of Fig. 1. We should note that our simulation resolution at the Earth's location is $3.44 R_{\odot}$, which corresponds to about 40 minutes for the solar wind data. Also, the timing given here is based on the steady-state solution using synoptic (27-day) magnetograms. We believe these two effects explain in part the earlier arrival of the DS at Earth as predicted from our solar wind model. This leads us to believe that the DS is real and is associated with the density enhancement on the TS at the location of the third structure. However, as noted above, the pink ray intersects not only this DS but also the second CME.

The analyzed HI-2 LOS image is a running difference; therefore, a dense region along the pink ray will corre- 

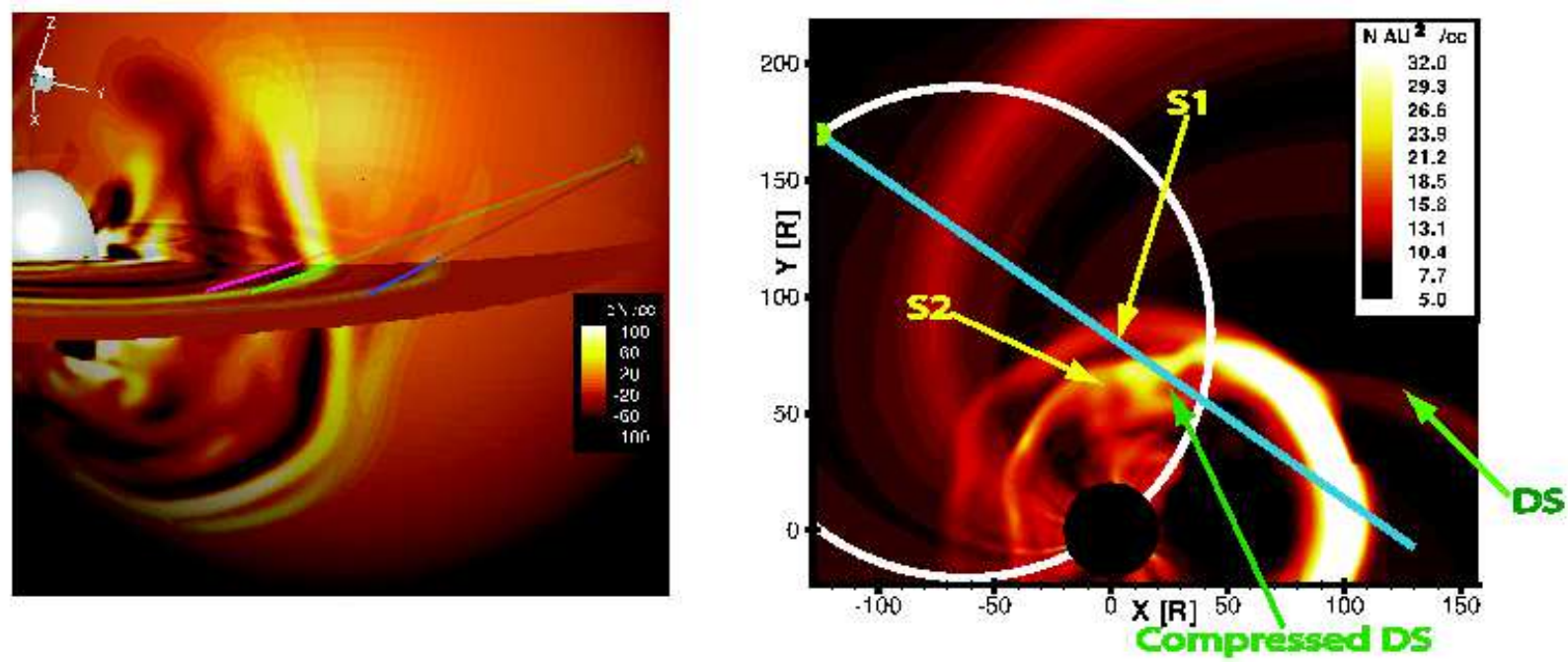

FIG. 2.- Left: 3-D view of the CMEs at 0600UT on 2007 January 26 showing the density increase (dN) with respect to 0400UT. The silver and green spheres depict the Sun and STEREO-A. The largest sphere is the TS. The three rays illustrate the LOS integration and correspond to the dots of the same color in the previous Figure. In the online version, the khaki and brown isosurfaces are regions of dN $= \pm 80 \mathrm{~cm}^{-3}$ respectively. Right: 2 -D cut at 0600UT on January 26 as seen from solar north pole showing the density scaled by $1 / \mathrm{R}^{2}$. The structures corresponding to the two CMEs are indicated with the S1 and S2 arrows. The co-rotating dense stream before (resp. after) being hit by the CME fronts is indicated with the DS (resp. "compressed DS") arrow. The Sun, STEREO-A and the TS are represented by the black disk, the green disk and the white circle respectively. The blue line delimits the inner FOV of HI- $2 \mathrm{~A}, 20^{\circ}$ away from the Sun.

spond to a bright front at the pink dot only if no dense region was present there in the previous image. In fact, the pink ray also intercepted the CME front in the previous frame, whereas it did not intersect the DS. Hence, the CME front is not expected to contribute to the brightness enhancement in the HI-2 image. Accordingly, we believe that the brightness enhancement, in the running difference image, is due to the DS, which was compressed by the two CMEs.

To summarize, there are three structures seen in the synthetic LOS image: one corresponding to the leading CME having not yet been overtaken by the following CME on the TS (blue dot); a second one corresponding to the following CME, or the merged CMEs away from the TS (green dot); and a third one resulting from the compression of the co-rotating DS by the CMEs (pink dot)

Careful analyses of HI-2 images with the support of 3D simulations such as the one presented here should help develop a better understanding of which density structures at which position are tracked by STEREO HIs. This, in turn, will help increase the accuracy of space weather predictions based on STEREO observations.

\section{DISCUSSION AND CONCLUSIONS}

In this Letter, we have discussed some apparent difficulties associated with the interpretation of STEREO HI-2 images, especially when there are multiple density structures in the field-of-view of the instrument. By means of a fully 3-D MHD simulation of the 2007 January 24-25 ejections, we have been able to associate the observed HI- 2 features with features seen in the synthetic HI-2 images. We have found that they correspond to the front associated with the leading CME, the front associated with the newly merged CMEs, and a co-rotating DS compressed by the two ejections. We believe that the observations could have been misinterpreted without the aid of a numerical simulation. This is because the DS appearance and evolution could have been mistaken for those of a CME. This work emphasizes the importance of carrying out 3-D simulations of CME interaction with a structured solar wind in interpreting STEREO observations. It should also be remembered that, without carefully considering the effect of subtracting consecutive images, one might not be certain with which structure (and which part of a structure) a bright front is associated. This is because most observational rays intersect multiple density structures in the heliosphere. In future related studies, we will present a detailed comparison of the simulated and real SECCHI images for the 2007/01/24-25 events, thus filling the 20-hour SECCHI data gap.

The simulation reported here was carried out on a dedicated cluster of the solar group at the IfA. The research for this manuscript was supported by NSF grants ATM0639335 and ATM0819653 and NASA grants NNX07AC13G and NNX08AQ16G. W. M. was supported by NASA grant NNX06AC36G. We would like to thank the reviewer for helping us improve and clarify this Letter. The SECCHI data is produced by an international consortium of the NRL, LMSAL and NASA GSFC (USA), RAL and U. Bham (UK), MPS (Germany), CSL (Belgium), IOTA and IAS (France). SOLIS data were obtained from NSO.

\section{REFERENCES}

Arge, C., \& Pizzo, V. 2000, J. Geophys. Res., 105, 10465 Aschwanden, M. J., et al. 2008, Space Science Rev., 136, 565
Cohen, O., et al. 2007, ApJL, 654, L163

Eyles, C. et al. 2000, ApJ, 217, 319 
Farrugia, C. J. et al. 2006, J. Geophys. Res., 111, 11104

Harrison, R., Davis, C., \& Eyles, C. 2005,

Adv. Space Res., 36, 1512

Harrison, R., et al. 2008, Sol. Phys., 247, 171

Howard, R. A. et al. 2008, Space Science Rev., 136, 67

Jackson, B. V., \& Leinert, C. 1985, J. Geophys. Res., 90, 10759

Kaiser, M. L. et al. 2008, Space Sci. Rev., 136, 5

Lugaz, N., Manchester, W., Gombosi, T. 2005, ApJ, 627, 1019

Lugaz, N.,et al. 2007, ApJ, 659, 788

Lugaz, N. et al. 2008, J. of Atm. and Terrest. Phys., 70, 598

Manchester, W. B. et al. 2008, ApJ, 684, Sept. 10, in press

Minnaert, M. 1930, Zeitschrift fur Astrophysik, 1, 209

Odstrcil, D., Pizzo, V., \& Arge, C. 2005, J. Geophys. Res., 110, 2106
Rouillard, A. et al. 2008, Geophys. Res. Lett., in press Roussev, I. I. et al. 2003, ApJ, 588, L45

Roussev, I. I., \& Sokolov, I. V. 2006, ed. N. Gopalswamy,

R. Mewaldt, and J. Torsti, Geophys. Monog. 165, 89, 165, 89

Schwenn, R., et al. 2006, Space Science Rev., 123, 127

Sheeley, N. R., et al. 2008a, ApJL, 674, L109

Sheeley, N. R., et al. 2008b, ApJ, 675, 853

Sun, W., et al. 2008, Space Weather, 6, S03006

Titov, V. S., \& Démoulin, P. 1999, A\&A, 351, 707

Tóth, G., et al. 2005, J. Geophys. Res., 110, 12226

Vourlidas, A., \& Howard, R. A. 2006, ApJ, 642, 1216

Wang, Y.-M., \& Sheeley, N. R., Jr. 1990, ApJ, 355, 726 